\title{
Special Issue on: Airborne Simultaneous Localisation and Map Building (A-SLAM)
}

\author{
Nabil Aouf • Anibal Ollero • Jurek Z. Sasiadek
}

Published online: 3 April 2009

(C) Springer Science + Business Media B.V. 2009

Aerial robots can be considered as the natural evolution of the Unmanned Aerial Vehicles (UAVs) from the point of view of the intelligence. Traditional industrial robots evolved from the repetition of sequences of previously defined way points, with minimal perception and decision capabilities, toward robots interacting with the environment, mapping unknown environments and localising the robot in these maps. Thus, Simultaneous Localisation and Mapping (SLAM), has played an important role in the exploration of unknown environments. In the same way, Unmanned Aerial Vehicles will also evolve from the current commercial UAVs that mainly move by following a list of way points, to aerial robots capable of perceiving the environment adapting their behaviour to dynamic events. In addition, current UAV navigation strongly depends on satellite positioning systems, and then is sensitive to GPS transient failures, which are common in many outdoor environments. Furthermore, the navigation in GPS denied environments requires positioning devices that could be very costly and/or difficult to be implemented. Therefore, positioning with respect to the perceived environment is a need to increase the reliability or even to make possible UAV navigation. Moreover, environment mapping is also a very desirable feature for many UAV applications. Consequently, simultaneous

\footnotetext{
N. Aouf $(\varangle)$

Department of Informatics and Sensors, Cranfield University, Swindon, SN6 8LA, UK

e-mail: n.aouf@cranfield.ac.uk
}

\section{A. Ollero}

Robotics, Vision and Control Group Escuela Superior de Ingenieros, Universidad de Sevilla, Camino de los Descubrimientos, 4192 Seville, Spain e-mail: aollero@cartuja.us.es

\section{J. Z. Sasiadek}

Department of Mechanical and Aerospace Engineering, Carleton University, 1125 Colonel By Drive, Ottawa, K1S 5B6, Canada e-mail: jsas@connect.carleton.ca 
localisation and mapping is also becoming an important functionality in aerial robotics, as it was recognized years ago in ground robots.

This special issue is particularly devoted to simultaneous localisation and mapping in aerial robotics. It includes five papers which topics are related to several important topics in this new area ranging from new theoretical issues to practical implementations.

The first paper presents an algorithm that merges FastSLAM and the RaoBlackwellized particle filter to deal with state vector of higher dimension. The paper presents results using experimental data from an unmanned helicopter fusing measurements from on-board inertial sensors (accelerometer and gyro) and vision in order to solve the slam problem.

The second paper, from the University of Sydney, presents multi-vehicle active SLAM control architectures that actively plan the trajectories and motions of each of the vehicles based on maximising information in the localisation and mapping estimates in GPS denied environments where the UAVs use inertial sensor and terrain sensor information to simultaneously localise the UAV while building a point feature map of the surrounding terrain. The paper compares an ideal, centralised architecture, and decentralised architecture, where UAVs make their own control decisions based on common shared map information. Results are presented using a three-UAV team in a six-degree of freedom multi-UAV simulator.

The third paper, from the Madrid Polythecnical University, deals with Visual SLAM (VSLAM) by using images taken from UAVs in partially structured environments. The paper analyses several characteristics of the vision system to obtain more accurate localization and mapping from UAVs flights. The proposed system has been tested using the COLIBRI mini UAV by comparing the obtained results with the GPS information.

The fourth paper, from the University of Seville, uses mosaics to create a consistent view of the environment of the UAV and hence, to detect the drift in position estimation by using the mosaic as a resource. A method to build an stochastic mosaic given the image-to-image homographies is detailed. This technique is especially suitable for monitoring and surveillance tasks in which the UAV will repeatedly cover the same area. The paper also shows experimental results with real UAVs showing the benefit of the proposed method.

The last paper, from Cranfield University, presents a robust airborne 3D VSLAM based on a stereovision system. The method uses data fusion by means of nonlinear Hinfinity filtering scheme that avoids problems of the classical Extended Kalman Filtering techniques. A consistency and observability analysis for the Airborne VSLAM is also presented. Moreover, the paper presents a new approach to map management, based on the k-nearest landmark concept, that allows efficient loop closure detection and map building reducing the complexity of the VSLAM, which becomes independent of the map landmark number. Results show the efficiency of the proposed Airborne VSLAM solution. 